\title{
Predicting the Risk of Lymphedema in Breast Cancer Patients by Using Data Mining Techniques
}

\author{
Maliheh Fazeli ${ }^{1}$, Aliyeh Kazemi ${ }^{2}$, Shahpar Haghighat ${ }^{3,}$ \\ ${ }^{1}$ Department of Industrial Management, Faculty of Management, University of \\ Tehran, Tehran, Iran \\ ${ }^{2}$ Department of Industrial Management, Faculty of Management, University of \\ Tehran, Tehran, Iran \\ 3 Breast Cancer Research Center, Motamed Cancer Institute of ACECR, Tehran, Iran \\ * Corresponding author: Shahpar Haghighat, Breast Cancer Research Center, Motamed \\ Cancer Institute of ACECR, Tehran, Iran.E-mail: sha_haghighat@yahoo.com
}

DOI: $10.21859 /$ mci-supp-83

\section{Keywords:}

Lymphedema

Breast Cancer

Data Mining

Prediction

Neural Networks

Support Vector Machine

\begin{abstract}
Introduction: Data mining may be a great method for identifying the factors correlated with different outcomes. The purpose of this study is presenting important risk factors of occurrence lymphedema after breast cancer.

Materials and Methods: Data were collected from patients referring to the Seyed Khandan lymphedema clinic during 2009-2017. Data analysis was performed by using IBM SPSS Modeler software version 18 and CRISP-DM methodology. In the modeling section, neural networks (NNs) and support vector machine (SVM) methods were used.

Results: After data preparation, 933 data including 25 variables were selected as inputs of the models. Factors related to Lymphedema in patients with breast cancer were predicted and the probability of catching of Lymphedema in breast cancer patients was predicted by using NNs and SVM techniques. Sensitivity and accuracy of the algorithms to predict lymphedema probability showed $82.87 \%$ and $77.49 \%$ by SVM vs. $79.37 \%$ and $72.41 \%$ for NNs, respectively.

Considering SVM results, the most important risk factors of Lymphedema were heaviness, type of surgery, body mass index, ratio of the involved lymph node numbers to the removed lymph node numbers, radiotherapy, metastasis, patient's age at diagnosis, number of involved lymph nodes and occupation respectively.

Conclusions: Introducing lymphedema risk factors are important in preventive strategies. To pay attention to the patient' heaviness in arm should be considered as primary sign of lymphedema occurrence. Considering less invasive surgery and educating weight control to patients are recommended as preventive strategies of lymphedema.
\end{abstract}

